\title{
Um Sistema de Recomendação Baseado em um Modelo Cognitivo de Aprendizagem
}

\author{
André Prisco $\operatorname{Vargas}^{1}$, Rafael Santos ${ }^{1}$, Silvia Silva da Costa Botelho ${ }^{1}$, \\ Neilor Tonin ${ }^{2}$, Jean Bez ${ }^{3}$ \\ ${ }^{1}$ Universidade Federal do Rio Grande (FURG) \\ ${ }^{2}$ Universidade Regional Integrada do Alto Uruguai e das Missões \\ ${ }^{3}$ Universidade Federal do Rio Grande do Sul (UFRGS) \\ \{andre.prisco,rafaelpenna, silviacb\}@furg.br \\ \{neilor,bez\}@urionlinejudge.com.br
}

\begin{abstract}
Educational recommendation systems require different models from those applied in other areas. An important factor is recommend Learning $\mathrm{Ob}$ jects (LO) that improve the learning process and not only match with the student's personal preference. We used a game ranking technique, the ELO, to compare students and LO. The interaction of student and L.O. is like a game match. Analyzing the history of an online programming problem tool, we observed that recommending to the student problems that have ELO next to him tends to bring better results.
\end{abstract}

Resumo. Sistemas de recomendação para sistemas em educacionais demandam modelos diferentes dos utilizados em outras áreas. Um fator importante é apresentar objetos de aprendizagem (O.A.) que catalizem o aprendizado e não necessariamente operem em função dos gostos pessoais do estudante. Utilizando uma técnica de ranking em jogos, o ELO, comparamos estudantes e problemas como se os O.A. fossem duelos. Analisando o histórico de uma ferramenta online de problemas de programação, observamos que recomendar ao estudante problemas de ELO próximo ao seu tende a trazer melhores resultados.

\section{Introdução}

O problema de escolher, dentre diversos objetos, o mais adequado para um usuário é um problema já bastante trabalhado nos sistemas de recomendação. Sistemas de recomendação utilizam técnicas computacionais para selecionar itens de forma personalizada a usuários, levando em conta critérios como histórico e interesse. Esses sistemas têm sido construídos a fim de nos recomendar filmes,produtos ou sites mais adequados à nossa vontade. No entanto, o processo de recomendação na educação é diferente. Enquanto na escolha de filmes e sites podemos nos basear nos gostos do usuário e em seu histórico de utilização, a educação exige modelos específicos e uma análise diferente desses critérios, de forma que os objetos recomendados potencializem o aprendizado.

Os sistemas baseados em análise pedagógica geram sua recomendação através da modelagem de fundamentos pedagógicos, tornando assim a análise mais sofisticada no sentido de observar os dados sob a ótica de um modelo pedagógico. 
O objetivo deste trabalho é apresentar um sistema de recomendação de objetos de aprendizagem amparado em um modelo pedagógico. Mais especificamente adaptamos a arquitetura proposta em [Tang and McCalla 2005] para a criação de sistemas de recomendação educacionais. Neste artigo, focamos no desenvolvimento do modelo do estudante, que,baseado no modelo de aprendizagem de Piaget, procura potencializar a equilibração e o conflito cognitivo, trazendo uma motivação no aprendizado e tornando-o mais efetivo. A ideia central do módulo é relacionar os objetos que desafiem o estudante de modo adequado, sem que ele se entedie com problemas semelhantes nem se desmotive ao se deparar com problemas além de sua capacidade.

A epistemologia genética e os modelos pedagógicos relacionais compreendem a aprendizagem como a transformação tanto do sujeito (estudante) quanto do objeto a ser aprendido (em nosso caso, o objeto de aprendizagem e seu tema proposto) [Becker 2008, da SILVA 2011]. Como é na relação do objeto com o sujeito que ocorre a aprendizagem, fizemos um paralelo deste com os modelos aplicados em jogos, já que estes possuem técnicas para estudar as relações dos sujeitos-jogadores com os objetospartida ou objetos-adversários. Sistemas de match making em jogos eletrônicos têm por objetivo encontrar o melhor adversário para uma partida. Para um jogador que queira melhorar seu desempenho, ou seja, efetivar sua aprendizagem no jogo, as melhores partidas são aquelas em que ele pode melhorar sua técnica. Oponentes mais fracos são mais fáceis de vencer, mas oferecem pouca oportunidade de aprendizado, enquanto oponentes fortes demais podem ser desmotivadores ao jogador, que não tem o suporte para se apropriar de técnicas muito mais avançadas. Os sistemas então buscam encontrar adversários que potencializem o aprendizado. Adaptamos uma destas técnicas, o ELO, para auxiliar na recomendação, não de adversários, mas sim de desafios de programação para estudantes de computação. Utilizamos como estudo de caso um ambiente virtual de aprendizagem que possui um repositório com problemas de programação. Tal plataforma funciona através de um sistema de submissão, onde alunos enviam soluções algorítmicas para os problemas apresentados, recebendo feedback automático. O sistema é utilizado principalmente para treinos de maratonas de programação, mas também é aplicado em sala de aula em muitas instituições brasileiras e estrangeiras. Com milhares de estudantes e milhões de submissões registradas, a plataforma oferece a oportunidade de comparar diversos casos e comportamentos, através do histórico de cada estudante na plataforma.

\section{Arquitetura do Sistema de Recomendação Educacional}

Este trabalho se propõe a demonstrar a técnica de modelagem e diagnóstico da habilidade e engajamento do estudante, bem como a forma como a interação dos estudantes com os objetos de aprendizagem altera os metadados de tais objetos. Uma vez que tais técnicas estão inseridas no contexto de um sistema de recomendação, apresentamos brevemente a arquitetura do sistema, ainda que uma análise mais detalhada fuja do escopo deste trabalho. Os módulos da arquitetura estão ainda em processo de desenvolvimento e experimentação. A arquitetura foi baseada no sistema [Tang and McCalla 2003] e é apresentada na Figura 1.

O currículo é modelado através de rotas de aprendizagem [de Lima et al. 2016]. Abordar os conteúdos e objetos de aprendizagem em uma trajetória facilita um gerenciamento computacional e também fornece recursos para a criação da personalização do currículo, já que o modelo pode permitir diversas rotas, fornecendo para diferentes estu- 


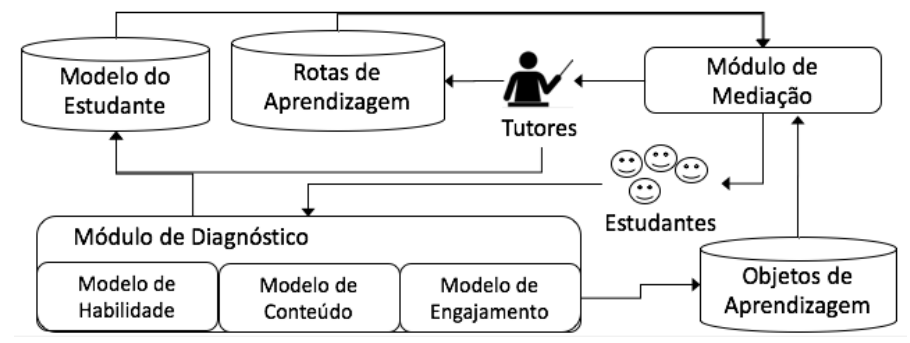

Figure 1. Arquitetura do Sistema de Recomendação

dantes diferentes experiências com objetos de aprendizagem e atividades didáticas mas gerando a mesma formação desejada. A rota de aprendizagem é modelada por um especialista no tópico e armazenado no módulo. No inicio do processo, os novos estudantes passam por uma avaliação diagnóstica junto ao tutor. Através deste há uma análise objetiva e subjetiva que instancia o estado atual do estudante dentro do modelo proposto (correspondendo às suas habilidades, conhecimentos e engajamento).

A base de Objetos de Aprendizagem é um repositório de objetos de aprendizagem digitais e híbridos ${ }^{1}$, que abrangem atividades digitais e presenciais, aplicadas no ensino híbrido [Bacich et al. 2015]. Os elementos dessa base possuem metadados relacionados ao tema de ensino e aos níveis de habilidade e engajamento ideais para os estudantes que vierem a interagir. Estes últimos são adaptados automaticamente ou semiautomaticamente utilizando nossa metodologia.

O Módulo de mediação analisa o modelo do estudante e as rotas de aprendizagem para escolher o melhor objeto de aprendizagem personalizado para cada estudante em seu contexto. A mediação pode ser feita orientando diretamente o estudante ou através de uma interface de interação com o tutor.

Através da análise da interação do estudante com o objeto de aprendizagem o gerador do modelo do estudante realiza um novo diagnóstico, ajustando as informações sobre seu conhecimento sobre o conteúdo, seus níveis das habilidades observadas e seu nível de engajamento. O resultado da análise também atualiza alguns metadados sobre o próprio objeto de aprendizagem, uma vez que a interação nos traz informações tanto do sujeito quanto do objeto a ser aprendido (observe seções 3.1 e 3.2). Dependendo do objeto, a avaliação pode ser automática ou semiautomática, com o auxílio do tutor.

Com o novo diagnóstico, o Módulo de Mediação analisa novamente quais são os novos passos do estudante na rota de aprendizagem, e qual objeto de aprendizagem será mais adequado para sua trajetória.

\section{Modelo do Estudante}

Nesta seção apresentamos como utilizamos elementos das técnicas de match making em jogos com a teoria cognitiva de Piaget para apresentar um modelo de estudante capaz de trabalhar seu engajamento e catalizar o processo de aprendizagem. Tal modelo é utilizado em grande parte para a tomada de decisão no Módulo de Mediação.

\footnotetext{
${ }^{1}$ Objetos híbridos são formados por recursos didáticos digitais que devem ser relacionados a roteiros a serem conduzidos presencialmente com professores e estudantes
} 
VI Congresso Brasileiro de Informática na Educação (CBIE 2017)

Anais do XXVIII Simpósio Brasileiro de Informática na Educação (SBIE 2017)

\subsection{Teoria Cognitiva de Piaget}

Nesta seção apresentamos, sob a abordagem cognitiva e mais especificamente da epistemologia genética de Piaget [Piaget 1972, Lefrançois 2012, Becker 1992], alguns conceitos que utilizamos no decorrer deste trabalho. O foco do nosso estudo está no período pós-formal de Piaget, que é o período que estuda a aprendizagem de adultos [Arlin 1975, Knight and Sutton 2004]. Apresentamos alguns conceitos:

- Esquema: O conhecimento são estruturas da mente que se relacionam a outras estruturas em um complexo sistema conexionista. Tais estruturas são denominadas de esquemas. São responsáveis pelo processamento de informação, busca de padrões, tomadas de decisões e etc.

- Assimilação: a aprendizagem se dá por um processo de adaptação e esta é viabilizada pelos processos de assimilação e acomodação. Quando o indivíduo se depara com um problema ou precisa interagir com o meio, ele lida com com este buscando esquemas que melhor se ajustem. A interação pode ser a leitura de um texto, dirigir um novo modelo de carro ou compreender um diferente problema de programação. Quando a mente encontra em seus esquemas uma forma de resolver ou interagir, dizemos que a mente assimilou o problema.

- Acomodação: Quando um problema não pôde ser assimilado pela mente, o indivíduo tem duas alternativas: desistir ou criar novos esquemas que preencham as lacunas. Essa construção e reconstrução de esquemas para assimilar novos problemas é chamada de acomodação.

- Equilibração: A tendência da mente humana de respeitar um equilíbrio entre a assimilação e a acomodação é denominada de equilibração. O indivíduo não se satisfaz somente assimilando os mesmos problemas mas também tem a tendência de se desmotivar ao se deparar com problemas que necessitem de uma acomodação mais drástica aos esquemas já construídos.

- Conflito Cognitivo: Quando a mente se depara com séries de problemas apresentados de modo a respeitar a sua equilibração, esta pode se colocar em um estado de motivação cognitiva no aprender, ou seja, a própria curiosidade ou a incomodação por não assimilar o problema, desperta o interesse na sua solução. [Lefrançois 2012].

Talvez uma das tarefas mais desafiadoras seja utilizar a tecnologia para reunir toda a informação que temos sobre cada estudante para que possamos apresentar caminhos que cooperem com este estado de motivação pela aprendizagem em si.

A seção a seguir apresenta algumas métricas de classificação de jogos, que, neste trabalho, são utilizadas para modelar os níveis de habilidade dos estudantes, através de uma abordagem cognitiva, possibilitando a percepção de seu aprendizado e a recomendação de objetos de aprendizagem adequados.

\subsection{Métricas de Classificação de Jogos e a Teoria Cognitiva}

O problema de escolher, dentre diversos objetos, o mais adequado para um usuário é um problema já bastante trabalhado nos sistemas de recomendação. Sistemas de recomendação têm sido construídos a fim de nos recomendar filmes, produtos ou sites mais adequados à nossa vontade. No entanto, o processo de recomendação na educação não segue as mesmas regras, conforme apontado por [Tang and McCalla 2005]. 
VI Congresso Brasileiro de Informática na Educação (CBIE 2017)

Anais do XXVIII Simpósio Brasileiro de Informática na Educação (SBIE 2017)

Enquanto na escolha de filmes podemos nos basear nos gostos do usuário, nem sempre devemos recomendar ao estudante o que ele mais quer, mas o objeto de aprendizagem mais adequado, ou seja, aquele que potencializa seu aprendizado e/ou seu ganho de habilidade no tema. Além disso, o feedback não é simplesmente a aceitação ou utilização do objeto, mas uma avaliação de toda a experiência do estudante com ele.

Segundo [Tang and McCalla 2005], os problemas específicos para um sistema de recomendação para educação são:

- Itens escolhidos pelos alunos podem não ser pedagogicamente apropriados;

- Personalização não deve ser feita apenas sobre a escolha dos objetos de aprendizagem, mas também sobre o feedback de suas utilizações;

- Os alunos não pretendem ler muitos artigos e textos.

Em [Klašnja-Milićević et al. 2011] são apresentados alguns ambientes de aprendizagem que buscam implementar sistemas de recomendação. [Krištofič 2005] e [Tang and McCalla 2005] propõem sistemas Web que indiquem as melhores navegações dos usuários, de forma que encontrem os objetos de aprendizagem mais adequados, levando em conta principalmente seus feedbakcs e históricos de navegação.

Um sistema de tutoria para ajudar os alunos a aprenderem programação é apresentado em [Klašnja-Milićević et al. 2011]. Protus foi projetado e implementado como um sistema de tutoria, capaz de recomendar materiais úteis e interessantes aos estudantes com base em seus diferentes conhecimentos, preferências, propósitos de aprendizagem e outras características. O sistema reconhece o estilo de aprendizagem do aluno e permite que ele siga por diferentes caminhos no desenvolvimento de atividades de aprendizagem. O algoritmo de mineração de padrões AprioriAll [Pi-lian 2005] é adotado para extrair padrões comportamentais (interação) a partir do registro das atividades feitas pelos alunos e recomendar os caminhos mais adequados de aprendizagem.

Muitos sistemas de recomendação para educação necessitam de métricas para classificar os objetos de aprendizagem e as habilidades dos estudantes, de forma que possam ser estabelecidas relações entre os alunos e as atividades mais adequadas para ele. No trabalho de [Tsai et al. 2006], um mecanismo de classificação adaptativo para personalização do ensino utilizando repositórios na Internet é apresentado. O sistema utiliza uma função de distância entre os usuários baseada em similaridade de interesses para classificar o grau de relevância dos objetos de aprendizagem de acordo com a intenção de um usuário.

Com relação a essas métricas de relacionamento de atividades e estudantes, as teorias desenvolvidas para jogos podem ser úteis. O match making é a tarefa que produtores de jogos e técnicos fazem para escolher o melhor oponente para um jogador [Davis et al. 2003]. Neste caso, o objetivo não é escolher o oponente que mais agrada, mas aquele que potencializa o ganho de habilidade e minimiza o risco de desmotivação. No caso do método criado por [Davis et al. 2003], jogadores são escolhidos para uma partida de forma que se tenha uma maior jogabilidade, levando em conta fatores como a linguagem, nível de habilidade e localização.

Um dos modelos mais utilizados para esta tarefa foi desenvolvido para torneios de Xadrez e hoje é utilizado em diversos esportes e jogos eletrônicos. O ELO visa quantificar os jogadores através do seus históricos de jogos. O ELO é um sistema de ranqueamento 
VI Congresso Brasileiro de Informática na Educação (CBIE 2017)

Anais do XXVIII Simpósio Brasileiro de Informática na Educação (SBIE 2017)

estatístico que consegue calcular valores de nível de habilidade relativa para competidores ou máquinas em jogos competitivos. A ideia do sistema de ranqueamento por ELO [Elo 1978] foi criada por um professor de física americano, nascido na Hungria, chamado Arpad Elo. O sistema ELO funciona da seguinte maneira:

- Cada jogador possui um valor de habilidade associado a si (ELO);

- A cada partida ocorrida, os ELOs dos jogadores envolvidos são atualizados;

- Antes da partida ocorrer, existe uma probabilidade calculada (baseada nos ELOs de cada um) do jogador 1 vencer o jogador 2 (e vice-versa);

- Após o jogo, caso a vitória ocorra por parte do jogador com maior probabilidade de vencer, os ELOs de ambos jogadores praticamente não sofrem alteração;

- Após o jogo, caso a vitória ocorra por parte do jogador com menor probabilidade de vencer, os ELOs de ambos são significativamente alterados, o vencedor tendo seu ELO aumentado e o perdedor diminuído;

A demonstração dos cálculos das probabilidades e das atualizações dos ELOs podem ser encontradas em [Elo 1961].

Para este trabalho, apresentamos a hipótese de que o processo de melhorar em um jogo é um processo de equilibração, apresentado no capítulo anterior. Ao escolher os jogadores ideais a se enfrentarem (match making), o ELO surge como um mecanismo facilitador para o processo de equilibração. Os jogadores possuem um esquema formado para lidar com o jogo. Ao enfrentar jogadores mais difíceis e com ELOs adequados, estes não possuem esquemas formados e necessitam passar pelas etapas de assimilação e acomodação, de maneira que adquiram novas habilidades (aumentando seus ELOs), chegando finalmente a um estado de equilibração. Nessas situações, acreditamos que os sujeitos se motivam e aprendem através da manipulação dos objetos adequados (partidas com certo grau de desafio) e do conflito cognitivo provocado. Dessa forma, esse tipo de jogo pode ser tratado como um processo de aprendizagem de Piaget e assim, a métrica de desempenho dos jogos pode ser um indicador para a avaliação da aprendizagem.

\section{Modelo de Habilidade em uma Plataforma de Ensino de Programação}

A fim de analisar o modelo de habilidade do estudante, desenvolvemos o experimento descrito a seguir. Utilizamos a plataforma de ensino de programação URI Online Judge ${ }^{2}$, na qual estudantes de computação tentam resolver problemas de programação. A plataforma possui um repositório de objetos de aprendizagem, os quais possuem um formato específico com os seguintes dados: nome do problema; número do problema; tempo de execução máximo; nível de dificuldade do problema; categoria do problema; textos e imagens explicativas sobre problema a ser resolvido; uma explicação e um exemplo de como deve ser a entrada de dados; uma explicação e um exemplo de como deve ser a saída de dados;

Além disso, a manipulação sobre estes OA também são particulares:

- os alunos devem fazer um programa em uma linguagem de programação para resolver o problema proposto no OA;

- após, o aluno deve submeter sua solução à plataforma para ser avaliado;

\footnotetext{
${ }^{2}$ URI online Judge é uma ferramenta utilizada em diversas universidades no país e no mundo. Pode ser acessada em https://www.urionlinejudge.com.br
} 
VI Congresso Brasileiro de Informática na Educação (CBIE 2017)

Anais do XXVIII Simpósio Brasileiro de Informática na Educação (SBIE 2017)

- a plataforma possui um juiz automático que analisa as respostas do programa submetido pelo aluno e dá um feedback;

- o feedback pode ser: Accepted, quando o problema foi aceito sem nenhum erro; Compilation error, quando alguma estrutura do código foi escrita de maneira errada; Possible runtime error, quando ocorre um possível erro no fluxo de execução do programa; Presentation error, quando os dados de saída não estão formatados conforme descrito enunciado do problema; Runtime error, quando existe um erro no fluxo de execução do programa; Time limit exceeded, quando o tempo de execução excedeu o estipulado no enunciado do problema; Wrong answer, quando o programa executou normalmente, porém, apresentou saídas incorretas.

Atualmente a plataforma conta com mais de 1500 problemas, mais de 70 mil usuários e mais de 4,5 milhões de submissões. Os principais usuários da plataforma são estudantes que submetem suas soluções aos problemas de programação como atividade avaliada em seus cursos, por estudantes que querem praticar de forma independente e por estudantes que treinam para maratonas de programação. Estes são livres para escolher os problemas que querem resolver. Cabe salientar que, neste trabalho, queremos avaliar o modelo de habilidade do estudante e não o sistema de recomendação como um todo. Sendo assim, optamos por observar o histórico da plataforma, no qual os estudantes escolheram livremente os problemas, e observamos aqueles que seguiram ou não as diretrizes que daríamos se o sistema tivesse feito as recomendações.

Em nosso experimento, a análise dos dados passa por uma modificação na utilização do sistema ELO que comentamos na introdução. Enquanto o ELO trata da relação de um jogador perante outro jogador, nossa abordagem adapta a métrica para relacionar o estudante ao problema a ser tentado. A epistemologia genética é relacional, ou seja, considera o aprendizado como uma relação do indivíduo com o objeto a ser aprendido. Dessa forma, atribuir ELOs aos problemas, bem como aos estudantes, e então compará-los é um modo elegante de modificar a métrica para se adequar ao nosso modelo.

Consideramos cada submissão como um jogo, um "duelo" entre o jogador e o problema. Como abordado por Piaget, a aprendizagem permite modificar e manipular o objeto a ser aprendido, na mesma medida que modifica o próprio indivíduo. Tal conceito é modelado a partir da variação dos ELOs dos estudantes e dos problemas. A cada inicio de submissão, nosso algoritmo tem armazenado o ELO do problema e do estudante. Um feedback positivo (accepted) indica que o estudante "venceu o jogo" enquanto um feedback negativo (wrong answer, timelimit e etc) indica que o "problema venceu". Após a avaliação, os ELOs são então atualizados. Cada problema é um objeto de aprendizagem que não tem seu conteúdo modificado com o tempo, portanto sua variação de ELO deve ser controlada. A seguir apresentamos como abordamos este problema.

A metodologia adotada segue conforme a Figura 2. Os dados coletados e armazenados em um SGBD (utilizamos o PostgreSQL ${ }^{3}$ ). No próprio banco de dados, fizemos tratamentos simples, como eliminação de dados replicados e transformação de dados textuais para dados numéricos (para facilitar o processamento). A seguir, atribuímos um ELO inicial para cada estudante e para cada problema. Nesta etapa inicial, todos os estudantes recebem um ELO médio (1100). Para atribuição do ELO dos problemas, utilizamos como heurística o nível de dificuldade indicado pelo autor do problema. Não há

\footnotetext{
${ }^{3}$ https://www.postgresql.org
} 


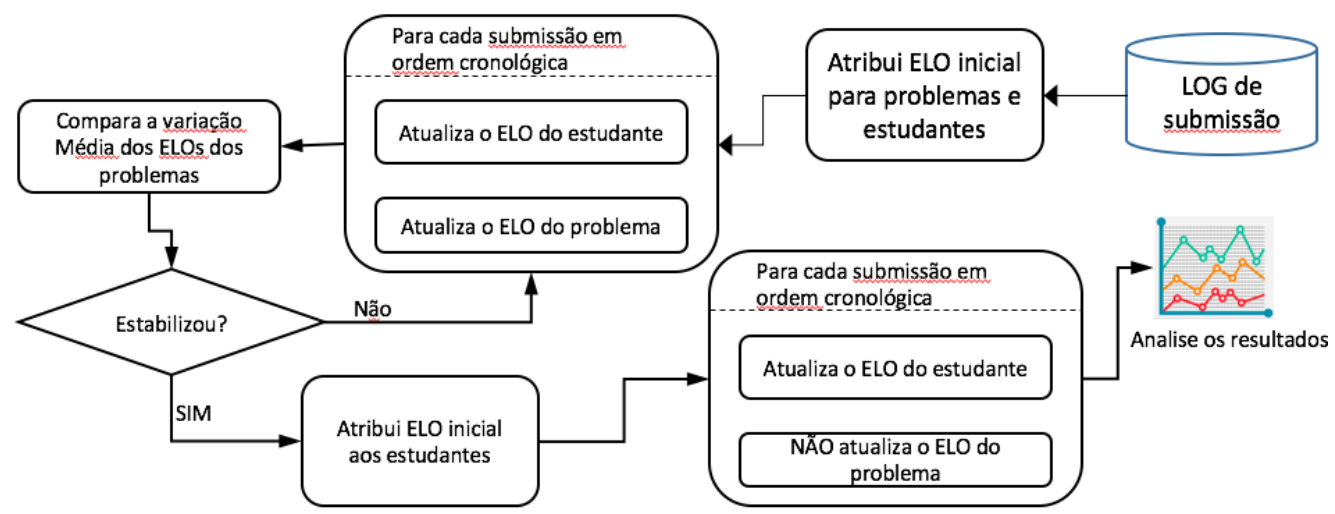

Figure 2. Metodologia adotada para o modelo de habilidade do estudante

necessidade de se atribuir com precisão um ELO inicial uma vez que o algoritmo iterativo fará o ajuste fino dos valores. No entanto, um bom valor inicial proporciona uma convergência mais rápida, o que facilita o experimento.

Com os ELOs iniciais atribuídos, nosso algoritmo simula cada submissão efetuada em ordem cronológica. Através do feedback de cada submissão, os ELOs são atualizados. Ao final do processo tem-se os ELOs finais de cada estudante e de cada problema. Compara-se então os valores ao final do processo com os valores anteriores ao processo. Se a diferença entre os valores é substancial isso indica que os ELOs ainda não convergiram e repete-se o processo. Assim que o processo converge, armazenamos os ELOs finais de cada problema e o consideramos constantes. Esta é outra modificação que fizemos na métrica ELO. Uma vez que cada problema é um objeto de aprendizagem que não tem seu conteúdo modificado com o tempo, a variação de ELO deve ser restrita à etapa de convergência. Uma vez conhecido o ELO de cada problema, consideramos que este seja constante para a próxima etapa.

Uma vez que a etapa de convergência foi terminada, os estudantes recebem novamente ELOs médios e para cada nova submissão seus ELOs são atualizados e armazenados na base de dados. Tem-se então o histórico do ELO de cada estudante para cada submissão. Com estes dados, pudemos começar as análises apresentadas na seção 5.

\section{Resultados e Conclusão}

Após a aplicação da metodologia, em um primeiro momento, analisamos o histórico do ELO de alguns estudantes para cada submissão (Figura 3). Neste caso, entendemos que o aluno passa por uma fase de adequação (em que seu ELO baixa), até que, em certo momento, ele encontra os problemas adequados, fazendo com que seu ELO comece a subir.

Posteriormente, analisamos os resultados separadamente em 2 grupos. No primeiro grupo, foram observadas todas as submissões em que a habilidade do aluno parecesse ser adequada ou superior para resolver o problema escolhido, ou seja, problemas com ELOs menores ou ligeiramente maiores que o do aluno. Já para o segundo grupo de análise, foram observadas todas as submissões em que a habilidade do aluno parecesse ser inadequada para o problema escolhido, ou seja, problemas com ELO significativamente superior ao do aluno. A Tabela 1 apresenta os resultados dessas análises. 
VI Congresso Brasileiro de Informática na Educação (CBIE 2017)

Anais do XXVIII Simpósio Brasileiro de Informática na Educação (SBIE 2017)

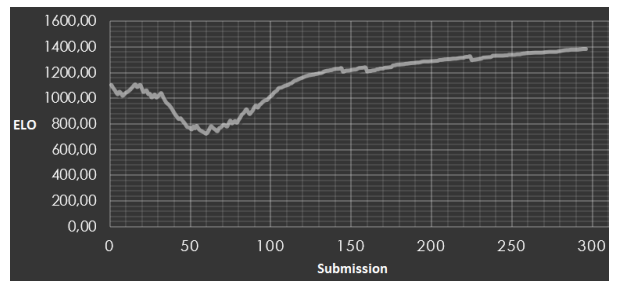

Figure 3. Histórico do ELO de um Estudante

\begin{tabular}{|l|l|l|l|}
\hline Grupo de Análise & Nro de submissões & Accepted & Error \\
\hline ELOs Adequados & 2,1 milhões & $61 \%$ & $39 \%$ \\
\hline ELOS Inadequados & 2,7 milhões & $38 \%$ & $62 \%$ \\
\hline
\end{tabular}

Table 1. Análise da aprendizagem nas submissões

Verificou-se, em um primeiro momento, que, das 4,8 milhões de submissões da base de dados, 2,1 milhões foram classificadas como submissões adequadas, isto é, o aluno escolheu um OA que parece ser ideal, com ELO semelhante ao seu e capaz de provocar conflito cognitivo e que o motive. Por outro lado, 2,7 milhões dos OA escolhidos pelos alunos foram classificados como inadequados, com ELO significativamente diferente do seu, parecendo ser ou muito difícil ou desmotivante.

Das 2,1 milhões de submissões consideradas adequadas, $61 \%$ obtiveram a resposta Accepted, isto é, os alunos acertaram o problema. Enquanto que dessas mesmas submissões, $39 \%$ obtiveram algum tipo de erro.

Em relação ao segundo grupo de análise, das 2,7 milhões de submissões consideradas inadequadas, $38 \%$ obtiveram a resposta Accepted, isto é, os alunos acertaram o problema. Enquanto que dessas mesmas submissões, $62 \%$ obtiveram algum tipo de erro.

Os resultados apontam que, em geral, quando os estudantes escolhem problemas mais adequados (ELOs semelhantes ou inferiores aos seus), eles obtém um maior número de acertos em suas submissões. Já quando a escolha é feita por problemas que não parecem ser desafiadores ou desmotivantes, em geral, eles erram mais ou acabam por desistir da aprendizagem na plataforma.

Por fim, cabe ressaltar que a observação e análise de dados na educação (Learning Analytics) é uma área vasta e muito promissora. Utilizar a área de sistemas de recomendação pode ajudar muito o entendimento do perfil do estudante e personalizar o ensino, respeitando as peculiaridades de cada aluno. As análises feitas para este trabalho representam os primeiros passos nessa direção. Há muito a ser feito, considerando outras informações não exploradas. Outras métricas como TrueSkill [Dangauthier et al. 2007] estão sendo implementadas e pretende-se apresentar seus resultados em um próximo trabalho, bem como uma análise aprimorada em dados advindos de um sistema de recomendação real. Acreditamos que novos resultados trarão informações úteis para professores e estudantes.

\section{References}

Arlin, P. K. (1975). Cognitive development in adulthood: A fifth stage? Developmental psychology, 11(5):602. 
VI Congresso Brasileiro de Informática na Educação (CBIE 2017)

Anais do XXVIII Simpósio Brasileiro de Informática na Educação (SBIE 2017)

Bacich, L., Neto, A. T., and de Mello Trevisani, F. (2015). Ensino híbrido: personalização e tecnologia na educação. Penso Editora.

Becker, F. (1992). O que é construtivismo. Revista de educação AEC, Brasília, 21(83):715.

Becker, F. (2008). Modelos pedagógicos e modelos epistemológicos. Metodologia: construção de uma proposta científica. Curitiba: Camões, pages 45-56.

da SILVA, J. A. (2011). Repetição e desafio nos exercícios escolares: dois lados de uma mesma moeda. Schème-Revista Eletrônica de Psicologia e Epistemologia Genéticas, 1(1):95-107.

Dangauthier, P., Herbrich, R., Minka, T., Graepel, T., et al. (2007). Trueskill through time: Revisiting the history of chess. In NIPS, pages 337-344.

Davis, J., Feldman, M., and Johansen, B. (2003). System and method for combining automatic opponent matching for computer gaming with chat room searchers. US Patent App. 10/637,048.

de Lima, J. V., Nunes, F. B., Zunguze, M. C., and Canto, A. B. (2016). Trajetórias de Aprendizagem.

Elo, A. (1961). New uscf rating system. Chess Life, 16:160-161.

Elo, A. E. (1978). The rating of chessplayers, past and present. Arco Pub.

Klašnja-Milićević, A., Vesin, B., Ivanović, M., and Budimac, Z. (2011). E-learning personalization based on hybrid recommendation strategy and learning style identification. Computers \& Education, 56(3):885-899.

Knight, C. C. and Sutton, R. E. (2004). Neo-piagetian theory and research: enhancing pedagogical practice for educators of adults. London Review of Education, 2(1):47-60.

Krištofič, A. (2005). Recommender system for adaptive hypermedia applications. In IIT. SRC 2005: Student Research Conference, page 229.

Lefrançois, G. R. (2012). Theories of human learning: What the professor said. Cengage Learning.

Pi-lian, W. T. H. (2005). Web log mining by an improved aprioriall algorithm. Engineering and Technology, , 4(2005):97-100.

Piaget, J. (1972). Intellectual evolution from adolescence to adulthood. Human development, 15(1):1-12.

Tang, T. Y. and McCalla, G. (2003). Smart recommendation for an evolving e-learning system. In Workshop on Technologies for Electronic Documents for Supporting Learning, International Conference on Artificial Intelligence in Education, pages 699-710.

Tang, T. Y. and McCalla, G. (2005). Smart recommendation for an evolving e-learning system: Architecture and experiment. International Journal on elearning, 4(1):105.

Tsai, K. H., Chiu, T. K., Lee, M. C., and Wang, T. I. (2006). A learning objects recommendation model based on the preference and ontological approaches. In Advanced Learning Technologies, 2006. Sixth International Conference on, pages 36-40. IEEE. 